Pacific Journal of Mathematics

ORDER-NDDCEE TOPOLOGICAL PROP 


\title{
ORDER-INDUCED TOPOLOGICAL PROPERTIES
}

\author{
Susan J. Andima ${ }^{1}$ And W. J. Thron
}

Each topology $\mathscr{T}$ on a set $X$ may be associated with a preorder relation $R_{\mathscr{F}}$ on $X$ defined by $\langle a, b\rangle \in R_{\mathscr{T}}$ iff every open set containing $b$ contains $a$. Although the correspondence is many-to-one, there is always a least topology, $\mu(R)$, and a greatest topology, $\nu(R)$, having a given preorder $R$. This leads to a natural correspondence between order properties and some topological properties and to the concept of an order-induced topological property. We show that a number of familiar topological properties (mostly lower separation axioms) are order-induced and also consider some new properties suggested by order properties. Let $T_{p}$ be an order-induced topological property with associated order property $K_{p *}$ We characterize minimal and maximal $T_{p}$ as follows: A topological space $(X, \mathscr{T})$ is maximal $T_{p}$ iff $\mathscr{T}=\nu\left(R_{\mathscr{F}}\right)$ and $R_{\mathscr{T}}$ is minimal $K_{p \cdot}$. With the imposition of a further condition on the class $K_{p}$ (satisfied by most properties under discussion), $(X, \mathscr{T})$ is minimal $T_{p}$ iff $\mathscr{T}=\mu\left(R_{\mathscr{T}}\right)$ and $R_{\mathscr{T}}$ is maximal $K_{p}$. We apply these general theorems to a number of order-induced properties and conclude with an example to show that, for two particular properties, $\mathscr{T}$ may be minimal $T_{p}$ even though $R_{\mathscr{F}}$ is not maximal $K_{p}$.

1. Introduction. Correspondences between topologies and preorders on $X$ similar to that assigning $R_{\mathscr{T}}$ to $\mathscr{T}$ have been described by several mathematicians. Ore in 1943 [14] associated with each closure operator on a fixed set $X$ a preorder relation which, for the topological closure operators, is exactly the same as $\boldsymbol{R}_{\mathscr{g}}$. Others have restricted their attention to the "principal" or "discrete" spaces in which arbitrary intersections of open sets are open. Linfield in his thesis [11] of 1925 studied principal topologies whose preorders were equivalence relations [see 7], and in 1935 both Alexandroff [1] and Tucker [20] described a oneto-one correspondence between $T_{0}$ principal topologies and partial orders. Destouches in 1937 drew on the work of Linfield and Alexandroff to study principal spaces in general [6], and Steiner in 1966 showed that the lattice of principal topologies is anti-isomorphic to the lattice of preorder relations on $X$ [16]. Alexandroff, Tucker, and Steiner all assigned the relation $R_{\mathscr{G}}^{-1}$ to $\mathscr{T}$, and Lorrain (1969) used both $R_{\mathscr{T}}^{-1}$ and $\boldsymbol{R}_{\mathscr{T}}$ to define functors from the category of principal spaces to the category of preordered sets [13].

\footnotetext{
${ }^{1}$ Formerly Susan J. Zimmerman.
} 
Our way of associating a preorder with each topology on $X$ is related to the classical concept of an "ordered topology" in which the smallest topology associated with a given preorder is the interval topology with sets of form $\sim\{y: x \leqq y\}$ and $\sim\{y: y \leqq x\}$ as a subbase. In our scheme, the smallest topology with preorder $R$ is the point closure topology, $\mu(R)$, for which sets of form $\sim\{y: x \leqq y\}$ and $X$ form a subbase. Thus the interval topology is the least upper bound of $\mu(R)$ and $\mu\left(R^{-1}\right)$, and $\mu(R)$ can be thought of as a one-sided interval topology. This relationship makes order-induced properties of some assistance in studying ordered topologies [see 19].

Most of the topological and order terminology is that of Thron [18] and Birkhoff [3]. All topological spaces $(X, \mathscr{T})$ and all preordered spaces $(X, R)$ will be assumed to have at least two elements. The definitions of preorder (or quasi-order), totally ordered preorder, partial order, chain, lattice, and of the inverse (or converse), $R^{-1}$, of a relation $R$ are those of Birkhoff. The diagonal relation on a set $X,\{\langle x, x\rangle: x \in X\}$, is denoted by $\Delta$. A partial order is complete iff every non-empty subset bounded above has a least upper bound. For any preorder $R$ on $X$, the symbols $a R b$ and $a \leqq b$ both mean $\langle a, b\rangle \in R$. Such terms as least upper bound, maximal, and cover are used only for partial orders and are defined in Birkhoff. In a partial order, $b$ is a successor for $a$ iff $a<b$ and whenever $a<x$ then $b \leqq x$. Predecessors are defined dually. Definitions of upper and lower bounds are the same for preorders as for partial orders.

The set of all preorder relations on a fixed, but arbitrary, set $X$ forms a lattice on $\mathscr{P}(X \times X)$ when ordered by set inclusion. For any two preorder relations $R$ and $S$ on $X$, the greatest lower bound and the least upper bound are, respectively, $R \wedge S=R \cap S$, and $R \vee S=\{\langle x, y\rangle$ : there is a finite sequence $x=x_{0}, \cdots, x_{n}=y$ of elements of $X$ such that $\left.\left\langle x_{t-1}, x_{i}\right\rangle \in R \cup S, i=1, \cdots, n\right\}$. Furthermore, $(R \vee S)^{-1}=\left(R^{-1}\right) \vee\left(S^{-1}\right)$. The set of all topologies on $X$ also forms a lattice under set inclusion. For any two topologies $\mathscr{T}_{1}$ and $\mathscr{T}_{2}$ on $X, \mathscr{T}_{1} \wedge \mathscr{T}_{2}=\mathscr{T}_{1} \cap \mathscr{T}_{2}$, and, if $\mathscr{B}_{1}$ and $\mathscr{B}_{2}$ are bases for $\mathscr{T}_{1}$ and $\mathscr{T}_{2}$, then $\left\{B_{1} \cap B_{2}: B_{1} \in \mathscr{B}_{1}\right.$ and $\left.B_{2} \in \mathscr{B}_{2}\right\}$ is a base for $\mathscr{T}_{1} \vee \mathscr{T}_{2}$.

With each topology $\mathscr{T}$ on $X$, there is associated a relation, designated $R_{\mathscr{T}}$ or $\rho(\mathscr{T})$, on $X$ defined by $\langle a, b\rangle \in R_{\mathscr{T}}$ iff every open neighborhood of $b$ contains $a$. $\quad R_{\mathscr{T}}$ is always a preorder relation, and the function $\rho$ is order-reversing in that $\rho\left(\mathscr{T}_{1}\right) \supseteq \rho\left(\mathscr{T}_{2}\right)$ whenever $\mathscr{T}_{1} \subseteq \mathscr{T}_{2}$. That $\rho$ is a mapping onto the set of preorders is a consequence of Theorem 1.1.

In a topological space $(X, \mathscr{T})$, one says that $x$ is separated from $y$ iff there is an open neighborhood of $x$ which excludes $y$. If $x$ is separated from $y$ and $y$ is separated from $x$, then $x$ and $y$ are said to be 
separated. It is clear from the definitions that $\langle a, b\rangle \in R_{\mathscr{g}} \Leftrightarrow b$ is not separated from $a$. Because of this correspondence, the four sets below, originally defined by Aull and Thron [2] in terms of separation, may be re-defined in terms of the relation $R_{\mathscr{T}}$ as follows. Let $\mathscr{T}$ be a topology on $X$ and let $a \leqq b$ iff $\langle a, b\rangle \in R_{\mathscr{g}}$. Then, for each $x \in X$, the closure of $x$ is $\{\bar{x}\}=\{y: x \leqq y\}$, the derived set of $x$ is $\{x\}^{\prime}=\{y: x<y\}$, the kernel of $x$ is $\{\hat{x}\}=\{y: y \leqq x\}$, and the shell of $x$ is $\{\hat{x}\}=\{y: y<x\}$. Note that $G=\bigcup\{\{\hat{x}\}: x \in G\}$ for any $G \in \mathscr{T}$, and $\{\hat{x}\}=\cap\{G: x \in G \in \mathscr{T}\}$ for any $x \in X$.

For a given preorder $R$ on $X$, there are, in general, many topologies $\mathscr{T}$ for which $\rho(\mathscr{T})=R$, but all of these topologies have exactly the same point-closures, point-derived sets, kernels, and shells. Therefore, given a preorder $R$, it makes sense to write $\{\bar{x}\}$ or $\{\hat{x}\}$ even though no topology is specified. When needed for clarity, such notation as $\{\hat{x}\}^{\mathscr{T}}$ or $\{\hat{x}\}^{R}$ will be used. The two topologies defined below are of key importance, because one is always the least and the other always the greatest topology in the set of topologies on $X$ with fixed relation $R$, as is shown in Theorem 1.1.

Definition. Let $R$ be a preorder on $X . \quad \mu(R)$, the point-closure topology of $R$, is the smallest topology on $X$ in which all sets $\{\bar{x}\}=$ $\{y:\langle x, y\rangle \in R\}, x \in X$, are closed. $\nu(R)$, the kernel topology of $R$, is the smallest topology on $X$ in which all sets $\{\hat{x}\}=\{y:\langle y, x\rangle \in R\}, x \in X$, are open.

$\left\{\sim\{\bar{x}\}^{R}: x \in X\right\} \cup\{X\}$ is clearly a subbase for the point-closure topology $\mu(R)$, and it is easy to show that $\left\{\{\hat{x}\}^{R}: x \in X\right\}$ is a base for the kernel topology $\nu(R)$.

THEOREM 1.1. A topology $\mathscr{T}$ on $X$ has preorder $R$ iff $\mu(R) \subseteq \mathscr{T} \subseteq$ $\nu(R)$. In particular, $\rho(\mu(R))=\rho(\nu(R))=R$.

Proof. Assume that $\mathscr{T}$ has relation $R$. For each $x \in X,\{\bar{x}\}^{R}=$ $\{\bar{x}\}^{\mathscr{T}}$, which is closed in $(X, \mathscr{T})$. Therefore, since $\mu(R)$ is, by definition, the smallest topology in which the sets $\{\bar{x}\}^{R}$ are closed, $\mu(R) \subseteq \mathscr{T}$. For any $G \in \mathscr{T}, G=\cup\left\{\{\hat{x}\}^{\mathscr{F}}: x \in G\right\}=\bigcup\left\{\{\hat{x}\}^{R}: x \in G\right\} \in \nu(R)$. That is, $\mathscr{T} \subseteq \nu(R)$

Conversely, let $\mathscr{T}$ be a topology such that $\mu(R) \subseteq \mathscr{T} \subseteq \nu(R)$. Then $\rho(\mu(R)) \supseteq \rho(\mathscr{T}) \supseteq \rho(\nu(R))$. For each $x \in X,\{\bar{x}\}^{\mu(R)} \subseteq\{\bar{x}\}^{R}$, because $\{\bar{x}\}^{R}$ is closed in $\mu(R)$. Therefore, $\langle x, y\rangle \in \rho(\mu(R)) \Rightarrow y \in\{\bar{x}\}^{\mu(R)} \subseteq$ $\{\bar{x}\}^{R} \Rightarrow\langle x, y\rangle \in R$. That is, $R \supseteq \rho(\mu(R))$. Let $\langle x, y\rangle \in R$ and let $G$ be any open neighborhood of $y$ in $\nu(R)$. Since $\left\{\{\hat{x}\}^{R}: x \in X\right\}$ forms a base for $\nu(R), \exists b \in X$ such that $y \in\{\hat{b}\}^{R} \subseteq G$. Then $\langle x, y\rangle \in R$ and $\langle y, b\rangle \in R \Rightarrow\langle x, b\rangle \in R \Rightarrow x \in\{b\}^{R} \subseteq G$. Therefore, $\langle x, y\rangle \in \rho(\nu(R))$, and $\rho(\nu(R)) \supseteq R$. Combining, we have $R \supseteq \rho(\mu(R)) \supseteq \rho(\mathscr{T}) \supseteq$ $\rho(\nu(R)) \supseteq R \Rightarrow \rho(\mu(R))=\rho(\mathscr{T})=\rho(\nu(R))=r$. 
THEOREM 1.2. Let $R$ be a preorder on $X . \quad \mu(R)=\nu(R)$ iff, for all $x \in X, \sim\{\hat{x}\}$ is the union of a finite number of point closures.

Proof. Let $\mu(R)=\nu(R)$ and let $a \in X$. If $\sim\{\hat{a}\}=\varnothing$, then it is the union of an empty collection of point closures. Assume $\sim\{\hat{a}\} \neq \varnothing$. Then, since $\sim\{\hat{a}\}$ is closed in $\nu(R)=\mu(R), \sim\{\hat{a}\}$ is the intersection of finite unions of point closures. That is, $\sim\{\hat{a}\}=\cap\left\{F_{\alpha}: \alpha \in \mathscr{A}\right\}$ where $\mathscr{A}$ is an index set and, for each $\alpha \in \mathscr{A}, F_{\alpha}$ is a finite union of point closures. Because $a \notin \sim\{\hat{a}\}$, there is a $\beta \in \mathscr{A}$ such that $a \notin F_{\beta}$. Clearly, $\sim\{\hat{a}\} \subseteq F_{\beta}$. Let $x \in F_{\beta}$. Then $x \in\{\bar{p}\} \subseteq F_{\beta}$ for some $p \in X$, and $p \leqq x$. If $x \leqq a$, then $p \leqq a$, and $a \in\{\bar{p}\} \subseteq F_{\beta}$, which is a contradiction. Therefore, $x \equiv a$ and $x \in \sim\{\hat{a}\}$. Thus $F_{\beta}=\sim\{\hat{a}\}$, and $\sim\{\hat{a}\}$ is the union of a finite number of point closures.

Conversely, assume that, for each $a \in X, \sim\{\hat{a}\}$ is the union of a finite number of point closures. Then $\sim\{\hat{a}\}$ is closed in $\mu(R)$, and hence $\{\hat{a}\}$ is open. That is, $\nu(R) \subseteq \mu(R)$. But by Theorem 1.1, $\mu(R) \subseteq \nu(R)$, and the two are equal.

If $R$ is a linear order, then $\sim\{\hat{a}\}=\{a\}^{\prime}$, and the union of a finite number of point closures is always a point closure. In this case, Theorem 1.2 reduces to $\mu(R)=\nu(R)$ iff, for all $x \in X,\{x\}^{\prime}$ is either empty or a point closure. In order terminology this becomes:

Corollary 1.3. Let $R$ be a linear order. $\mu(R)=\nu(R)$ iff every nonmaximum element has a successor.

The antiatoms or ultratopologies of the lattice of topologies on a set $X$, described by Teng-Sun Liu [12] and Fröhlich [8], are the topologies of form $\mathscr{P}(\sim\{a\}) \cup \mathcal{U}$ where $\mathscr{P}(\sim\{a\})$ is the power set of $X \sim\{a\}$ and $U$ is an ultrafilter on $X$ not containing $\{a\}$. If $\boldsymbol{U}$ is a principal ultrafilter, $\mathcal{U}(b)$, where $b \neq a$, then $\mathscr{P}(\sim\{a\}) \cup \mathcal{U}(b)$ is called a principal ultratopology and is denoted $\mathscr{T}(a, b)$. A topology $\mathscr{T}$ on $X$ is principal if $\mathscr{T}$ can be written as the intersection of principal ultratopologies or if $\mathscr{T}$ is the discrete topology, $\mathscr{P}(X)=\mathscr{T}(a, a)$. Steiner has shown [16] that under this definition a topology is principal iff arbitrary intersections of open sets are open, so that the principal spaces are actually the discrete spaces of Alexandroff.

It is clear from the definition of a principal ultratopology and of the function $\rho$ that, for any topology $\mathscr{T}$ on $X,\langle a, b\rangle \in \rho(\mathscr{T}) \Rightarrow \mathscr{T} \subseteq \mathscr{T}(b, a)$.

THEOREM 1.4. (a) For any preorder $R, \quad \nu(R)=\cap\{\mathscr{T}(y, x)$ : $\langle x, y\rangle \in R\}$.

(b) A topology $\mathscr{T}$ on $X$ is principal iff $\mathscr{T}=\nu(\rho(\mathscr{T}))$.

(c) $\nu$ is a lattice anti-isomorphism from the lattice of preorder relations onto the lattice of principal topologies. 
Proof. (a) Since $\rho(\nu(R))=R,\langle a, b\rangle \in R \quad$ iff $\quad \nu(R) \subseteq \mathscr{T}(b, a)$. Therefore, $\quad \nu(R) \subseteq \mathscr{T}(y, x), \quad \forall\langle x, y\rangle \in R, \quad$ and $\quad \nu(R) \subseteq \cap\{\mathscr{T}(y, x)$ : $\langle x, y\rangle \in R\} . \quad$ Let $\quad \mathscr{T}=\cap\{\mathscr{T}(y, x):\langle x, y\rangle \in R\} . \quad \nu(R) \subseteq \mathscr{T} \Rightarrow R \supseteq$ $\rho(\mathscr{T})$. If $\langle a, b\rangle \in R$, then $\mathscr{T} \subseteq \mathscr{T}(b, a) \Rightarrow\langle a, b\rangle \in \rho(\mathscr{T})$. Thus $R=\rho(\mathscr{T})$ and $\mathscr{T} \subseteq \nu(R)$ by Theorem 1.1. Therefore, $\nu(R)=$ $\cap\{\mathscr{T}(y, x):\langle x, y\rangle \in R\}$.

(b) Let $\mathscr{T}$ be any principal topology on $X$. Since arbitrary intersections of open sets are open, $\{\hat{x}\}=\cap\{G: x \in G\}$ is open in $\mathscr{T} \forall x$, and $\nu(\rho(\mathscr{T})) \subseteq \mathscr{T}$. Therefore, by Theorem 1.1, $\mathscr{T}=\nu(\rho(\mathscr{T}))$. Conversely, if $\mathscr{T}=\nu(\rho(\mathscr{T})), \mathscr{T}$ is principal by part (a).

(c) Let $R \subseteq S$. Then $\{\hat{x}\}^{R} \subseteq\{\hat{x}\}^{s}, \forall x \in X$. For $G \in \nu(S), G=$ $\cup\left\{\{\hat{x}\}^{s}: x \in G\right\} \supseteq \cup\left\{\{\hat{x}\}^{R}: x \in G\right\} \supseteq G \Rightarrow G=\cup\left\{\{\hat{x}\}^{R}: x \in G\right\} \in \nu(R)$. Therefore, $\nu(R) \supseteq \nu(S)$, and $\nu$ is order-reversing. Finally, since $\rho(\nu(R))=R \forall$ preorder $R$ and $\nu(\rho(\mathscr{T}))=\mathscr{T} \forall$ principal topology $\mathscr{T}, \nu$ is bijective.

$\mu$ is also a one-to-one function from the preorders onto the set of point-closure topologies, but, unlike the function $\nu, \mu$ is not orderreversing, as the following example illustrates.

EXAMPLE 1.5. Let $X=(-\omega) \cup\{a, b\}$ where $-\omega=\{0,1,-2, \cdots\}$ and $a$ and $b$ are two points not in $-\omega$. Let $S$ be the usual order on $-\omega$, let $\quad R=S \cup\{\langle a, b\rangle\} \cup \Delta$, and let $R^{*}=R \cup\{\langle a,-n\rangle: n \in \omega\} \cup$ $\{\langle b,-n\rangle: n \in \omega\}$. That is, $R$ leaves $a$ and $b$ unrelated to $-\omega$, while $R^{*}$ puts them in as lower bounds. Then $\{b\}$ is closed in $\mu(R)$ but not $\mu\left(R^{*}\right)$, and $\{b\} \cup(-\omega)$ is closed in $\mu\left(R^{*}\right)$ but not $\mu(R)$. Thus $\mu(R)$ and $\mu\left(R^{*}\right)$ are unrelated, even though $R \subseteq R^{*}$.

\section{Order-induced topological properties. The corre-} spondence, $\rho$, between topologies and preorders leads to a natural correspondence between order properties and certain types of topological properties. By a topological property we mean a class $T$ of topological spaces such that, whenever $(X, \mathscr{T}) \in T$, any homeomorphic image of $(X, \mathscr{T})$ is also in $T .("(X, \mathscr{T})$ is a $T$-space" or " $\mathscr{T}$ is a $T$-topology" means $(X, \mathscr{T}) \in T$, and we may also write $\mathscr{T} \in T$.) An order property is a class $K$ of preordered sets such that, whenever $(X, R) \in K$, any orderisomorphic image of $(X, R)$ is in $K$. A topological property $T$ is order-induced iff there is an order property $K$ such that $(X, \mathscr{T}) \in T$ iff $(X, \rho(\mathscr{T})) \in K$.

It is not difficult to show that any homeomorphism from $(X, \mathscr{T})$ to $(Y, \mathscr{U})$ is also an order isomorphism from $(X, \rho(\mathscr{T}))$ to $(Y, \rho(U))$. Furthermore, any order isomorphism from $(X, R)$ to $(Y, S)$ preserves the kernels and is therefore a homeomorphism from $(X, \nu(R))$ to $(Y, \nu(S))$. Consequently, if $K$ is any class of preorder relations and $T$ 
is the class of all topologies such that $\rho(\mathscr{T}) \in K$, then $T$ is a topological property if and only if $K$ is an order property. Thus every order property determines a topological property, and order properties can be used to suggest new topological properties.

The concept of duality for order properties carries over to the order-induced topological properties, and extensive use of this is made in characterizing such properties. This duality does not, however, extend to maximal and minimal order-induced topological properties, as we shall see in $\$ 3$.

Several standard order properties correspond to classical topological properties as indicated in Theorem 2.1. (We omit the proofs, all of which are trivial.) Parts (c) and (d) involve Alexandroff's Axiome Multiplicatif: "Any intersection of point closures which is nonempty is itself a point closure", and his Axioms of Dimension: "Any decreasing or increasing sequence of distinct point closures is finite" [1]. Part (b) was first proved by Ore [14] and part ( $\mathrm{g}$ ) is a combination of the work of Ore and Davis [5]. Davis defined $R_{0}$-spaces (also called $R$-spaces [4]) to be those in which closed sets are separated from the points they exclude, which is equivalent to the partition property that $\{\bar{x}\} \cap\{\bar{y}\}=\varnothing$ or $\{\bar{x}\}=\{\bar{y}\}$ for all $x, y \in X$. It is also clear from (g) that $(X, \mathscr{T})$ is $R_{0}$ iff $\{\bar{x}\}=\{\hat{x}\}$ for all $x \in X$ (called "autoreciproque" or self-dual by Destouches [6]) and iff $\{\{\hat{x}\}: x \in X\}$ is a partition of $X$.

THEOREM 2.1. Let $\mathscr{T}$ be a topology on $X$ and let $R=\rho(\mathscr{T})$.

(a) $(X, \mathscr{T})$ is a $T_{1}$-space iff $R$ is the diagonal relation $\Delta$.

(b) $(X, \mathscr{T})$ is $T_{0}$ iff $R$ is a partial order.

(c) $R$ is a complete partial order iff $(X, \mathscr{T})$ is a $T_{0}$-space satisfying Alexandroff's "Axiome Multiplicatif".

(d) $R$ is a partial order in which every chain is finite iff $(X, \mathscr{T})$ is a $T_{0}$-space satisfying Alexandroff's "Axioms of Dimension". Such spaces will be labeled $T_{F C}$-spaces. ordered.

(e) $\mathscr{T}$ is nested ( $G \subseteq H$ or $H \subseteq G$ for all $G, H \in \mathscr{T}$ ) iff $R$ is totally

(f) $\mathscr{T}$ is a nested $T_{0^{-}}$-topology iff $R$ is a linear order.

(g) $R$ is an equivalence relation iff $(X, \mathscr{T})$ is an $R_{0}$-space.

Definition. Let $R$ be a preorder relation on $X$ and let $x, y \in$ $X$. $x$ is connected to $y$ iff there is a finite sequence $x=x_{0}, \cdots, x_{n}=y$ such that $\left\langle x_{i-1}, x_{i}\right\rangle \in R \cup R^{-1}, i=1, \cdots, n$. The $R$-components of $X$ are the equivalence classes with respect to the relation " $x \approx y$ iff $x$ is connected to $y$ ". $R$ is a connected relation iff $x$ is connected to $y$ for all $x, y \in X$, and a topological space $(X, \mathscr{T})$ is $R$-connected iff $R_{\mathscr{T}}$ is a connected relation.

A topological space $(X, \mathscr{T})$ is connected iff every open cover satisfies 
the finite chain condition, and also iff every open cover satisfies the simple chain condition [17]. There are similar characterizations for topologies with connected relations.

Definition. A family $\mathscr{A}$ of subsets of $X$ satisfies the finite chain condition iff for all $A, B \in \mathscr{A}$ there is a finite sequence $A=A_{0}, \cdots, A_{n}=$ $B$ of sets in $\mathscr{A}$ such that $A_{i-1} \cap A_{i} \neq \varnothing, i=1, \cdots, n$. $\mathscr{A}$ satisfies the simple chain condition iff for all $a, b \in X$ there is a finite sequence (called a simple chain from $a$ to $b$ ) $A_{0}, \cdots, A_{n}$ of sets in $\mathscr{A}$ such that $a \in A_{0} \sim A_{1}, b \in A_{n} \sim A_{n-1}, \quad$ and $A_{\imath} \cap A_{1} \neq \varnothing$ iff $|i-j| \leqq 1, \quad i, j=$ $1, \cdots, n$.

THEOREM 2.2. A topological space $(X, \mathscr{T})$ is $R$-connected iff $\{\{\hat{x}\}: x \in X\}$ satisfies the finite chain condition, or, equivalently, iff $\{\{\hat{x}\}: x \in X\}$ satisfies the simple chain condition. Furthermore, $\{\{\hat{x}\}: x \in$ $X\}$ may be replaced with $\{\{\bar{x}\}: x \in X\}$ in either of the two characterizations.

Proof. That $\{\{\hat{x}\}: x \in X\}$ may be replaced by $\{\{\bar{x}\}: x \in X\}$ follows because " $R$ is connected" is a self-dual order property. We will prove only the second characterization. Let $R=\rho(\mathscr{T})$. Let $(X, \mathscr{T})$ be $R$ connected and let $a, b \in X$. If $a=b,\{\{\hat{a}\}\}$ is a simple chain from $a$ to $b$. Assume $a \neq b$. Then there is a finite sequence $a=x_{0}, \cdots, x_{n}=b$ such that $\left\langle x_{i-1}, x_{i}\right\rangle \in R \cup R^{-1}$ for all $i=1, \cdots, n$, and the sequence may be chosen to be of minimal length. All elements of this sequence are distinct, and $\left\langle x_{i-1}, x_{i}\right\rangle \in R \Leftrightarrow\left\langle x_{i}, x_{i+1}\right\rangle \in R^{-1}, i=1, \cdots, n-1$. Otherwise, the sequence can be shortened. Of the two integers $n-1$ and $n$, let $p$ be the odd one and let $q$ be the even one. If $\left\langle x_{0}, x_{1}\right\rangle \in R$, then $\left\{\hat{x}_{1}\right\},\left\{\hat{x}_{3}\right\}, \cdots,\left\{\hat{x}_{p}\right\}$ is a simple chain from $a$ to $b$. If $\left\langle x_{0}, x_{1}\right\rangle \in R^{-1}$, then $\left\{\hat{x}_{0}\right\},\left\{\hat{x}_{2}\right\}, \cdots,\left\{\hat{x}_{q}\right\}$ is a simple chain from $a$ to $b$. Therefore, $\{\{\hat{x}\}: x \in X\}$ satisfies the simple chain condition.

Conversely, assume that $\{\{\hat{x}\}: x \in X\}$ satisfies the simple chain condition and let $a, b \in X$. Then there is a simple chain of kernels, $\left\{\hat{x}_{0}\right\},\left\{\hat{x}_{1}\right\}, \cdots,\left\{\hat{x}_{n}\right\}$, from $a$ to $b$. Since $\left\{\hat{x}_{t-1}\right\} \cap\left\{\hat{x}_{i}\right\} \neq \varnothing$ for each $i=$ $1, \cdots, n$, there is an $a_{\imath} \in X$ such that $a_{t} \leqq x_{1-1}$ and $a_{t} \leqq x_{1}$. Then $a, x_{0}, a_{1}, x_{1}, a_{2}, x_{2}, \cdots, a_{n}, x_{n}, b$ is a finite sequence connecting $a$ to $b$ in $R$. Therefore, $R$ is connected and $(X, \mathscr{T})$ is an $R$-connected space.

$R$-connected is stronger than connected, because, if $(X, \mathscr{T})$ is $R$-connected, then $X=\cup\{\{\hat{x}\}: x \in X\}$ is the union of a family of connected sets satisfying the finite chain condition and is thus connected. It follows as a corollary that a principal topological space is connected iff it is $R$-connected, as has been shown by Steiner [16].

All but the last of the following Aull and Thron separation axioms [2] are order-induced. 
Definition. A topological space $(X, \mathscr{T})$ is a $T_{F}$-space iff, given a finite set $F$ and an $x \notin F$, either $x$ is separated from $F$ or $F$ is separated from $x . \quad(X, \mathscr{T})$ is a $T_{F F}$-space iff, given two disjoint finite sets $F_{1}$ and $F_{2}$, either $F_{1}$ is separated from $F_{2}$ or $F_{2}$ is separated from $F_{1} .(X, \mathscr{T})$ is a $T(\beta)$-space iff for all $x \in X\{x\}^{\prime}$ is degenerate (i.e., $\{x\}^{\prime}$ is a singleton or empty). $(X, \mathscr{T})$ is $T(\epsilon)$ iff intersections of derived sets of distinct points are degenerate, and is $T_{Y}$ iff intersections of closures of distinct points are degenerate. $(X, \mathscr{T})$ is a $T(\zeta)$-space iff for each $x \in X\{x\}^{\prime}$ is the union of a family of point closures, $\{\{\bar{y}\}: y \in Y\}$, such that any two distinct elements of $Y$ are separated. $(X, \mathscr{T})$ is a $T(\gamma)$-space iff each derived set is the union of disjoint point closures, and $(X, \mathscr{T})$ is a $T(\delta)$-space iff each nonempty derived set is a point closure. Finally, $(X, \mathscr{T})$ is $T_{D}$ iff each derived set is closed.

$T\left(\beta^{\prime}\right), T\left(\gamma^{\prime}\right), T\left(\zeta^{\prime}\right)$ and $T\left(\delta^{\prime}\right)$ are defined as the duals of $\beta, \gamma, \zeta$ and $\delta$, respectively. For example, $\mathscr{T}$ is a $T\left(\delta^{\prime}\right)$-topology iff each nonempty shell is a kernel. $T\left(\beta^{\prime}, 0\right)$, defined as $T\left(\beta^{\prime}\right)$ and $T_{0}$, is also equivalent to the condition $T_{Y S}$ that for all $x \neq y$ in $X,\{\bar{x}\} \cap\{\bar{y}\}$ is $\varnothing,\{x\}$, or $\{y\}$ [2]. $T_{F}, T_{F F}, T(\epsilon)$, and $T_{Y}$ are all self-dual.

$T(\delta)$ as defined here differs from the original [2] in which point derived sets could never be empty. $T(\zeta)$ was not among the original axioms, but is a natural generalization of $T(\gamma)$, and $T_{Y}$ had its origins with J. W. T. Youngs [21]. $\quad T_{D}$ is included here because of its relation to maximal nested (Theorem 3.9), but $T_{D}$ is not itself an order-induced property. To see this, it is easy to show that $T_{D}$ is equivalent to $T_{0}$ for principal topologies, so that the $T_{D}$ and $T_{0}$ topologies are associated with the same class of preorders, and there will be $T_{D}$ and non- $T_{D}$ topologies with the same relation. The axioms $T_{U D}$ and $T_{D D}$ [2] are also not order-induced, because, for principal topologies, they are equivalent to $T_{0}$ and $T_{Y S}$, respectively.

Aull and Thron have shown [2] that, in terms of derived sets and shells, a space is $T_{F}$ iff for all $x \in X,\{x\}^{\prime}$ consists of points $y$ such that $\{y\}^{\prime}=\varnothing$ (property $T(\alpha)$ ), and also that a space is $T_{F F}$ iff $\{x\}^{\prime}=\varnothing$ for all but at most one $x$ or $\{\hat{x}\}=\varnothing$ for all but at most one $x$. It is these characterizations which are used to derive parts (a) and (d) of Theorem 2.3.

A few additional order properties are used in Theorems 2.3 and 2.4. Let $R$ be a preorder on $X$. A totally ordered set with $n$ elements has length $n-1$, and the length of $(X, R)$ is the least upper bound of the lengths of all totally ordered subsets of $(X, R) .(X, R)$ is a pre-semiroot iff, for each $x \in X,\{y: x \leqq y\}$ is totally ordered by $R$. If, in addition, $R$ is a partial order, $(X, R)$ is a semiroot. A root is a semiroot with a greatest element. $(X, R)$ is upward directed iff each pair of elements of $X$ has an upper bound. A partial order in which each pair of elements has a least upper bound is an upper semilattice. The terms pre-semitree, 
semitree, tree, downward directed, and lower semilattice are defined dually.

The proofs of the characterizations in Theorems 2.3 and 2.4 are all straightforward and are therefore omitted.

THEOREM 2.3. Let $\mathscr{T}$ be a topology on $X$ and let $R=\rho(\mathscr{T})$.

(a) $(X, \mathscr{T})$ is a $T_{F}$-space iff $R$ is a partial order of length at most 1 .

(b) $(X, \mathscr{T})$ is a $T(\beta)$-space iff for each $x \in X\langle x, y\rangle \in R$ for at most one $y \neq x$, or, alternatively, iff $R$ is a pre-semiroot of length at most 1 .

(c) $(X, \mathscr{T})$ is $T(\beta, 0)\left(T(\beta)\right.$ and $\left.T_{0}\right)$ iff $R$ is a semiroot of length at most 1 .

(d) $(X, \mathscr{T})$ is $T_{F F}$ iff $X$ has at most one nonsingleton $R$-component and that component is a tree or root of length 1 .

(e) $(X, \mathscr{T})$ is $T(\epsilon)$ iff $R$ is such that any two distinct points have at most one upper bound in $X \sim\{x, y\}$, or, equivalently, such that any two distinct points have at most one lower bound in $X \sim\{x, y\}$. This in turn implies that the length of $R$ is at most 2.

(f) $(X, \mathscr{T})$ is $T_{Y}$ iff $R$ is such that any two distinct elements have at most one upper bound, or, equivalently, iff $R$ is such that any two distinct elements have at most one lower bound. This implies that $R$ is a partial order with length at most 1.

(g) $(X, \mathscr{T})$ is a $T(\zeta)$-space iff $R$ is a partial order such that whenever $a<b$ there is a cover $c$ of $a$ such that $c \leqq b$.

(h) $(X, T)$ is $T(y)$ iff $R$ is a partial order such that two unrelated elements cannot have both an upper and a lower bound, and whenever $a<b$ there is a cover $c$ of $a$ such that $c \leqq b$.

(i) $(X, \mathscr{T})$ is a $T(\delta)$-space iff $R$ is a partial order such that every nonmaximal element has a successor.

The characterizations of $T\left(\beta^{\prime}\right), T\left(\beta^{\prime}, 0\right)$ or $T_{Y S}, T\left(\zeta^{\prime}\right), T\left(\gamma^{\prime}\right)$, and $T\left(\delta^{\prime}\right)$ are the duals of the appropriate statements in Theorem 2.3. For example, $(X, \mathscr{T})$ is a $T\left(\zeta^{\prime}\right)$-space iff $\rho(\mathscr{T})$ is a partial order such that, whenever $a<b, b$ covers an element $c \in X$ such that $a \leqq c$.

THEOREM 2.4. Let $\mathscr{T}$ be a topology on $X$ and let $R=\rho(\mathscr{T})$.

(a) $R$ is a pre-semiroot iff, for all $a, b \in X,\{\hat{a}\} \cap\{\hat{b}\}$ is $\varnothing,\{\hat{a}\}$, or $\{\hat{b}\}$.

(b) $R$ is a semiroot iff $(X, \mathscr{T})$ is $T_{0}$ and for all $a, b \in X,\{\hat{a}\} \cap\{\hat{b}\}$ is $\varnothing$, $\{\hat{a}\}$, or $\{\hat{b}\}$.

(c) $R$ is upward directed (i) iff the intersection of any two point closures is nonempty, (ii) iff the intersection of any two nonempty closed sets is nonempty, and (iii) iff $X$ cannot be written as the union of two open proper subsets.

(d) $R$ is an upper semilattice iff $\mathscr{T}$ is $T_{0}$ and the intersection of any two point closures is a point closure. 
As the properties in Theorem 2.4 have no generally accepted topological names, we shall call them by their order types. Topological properties corresponding to pre-semitree, semitree, downward directed, and lower semilattice are the duals of the appropriate statements in Theorem 2.4. For example, a topology $\mathscr{T}$ is downward directed iff the intersection of any two kernels is nonempty. However, statements (ii) and (iii) of part (c) are not expressed in order terminology and cannot be dualized. In fact, no corresponding statements hold for a downward directed topology.

Pre-semitree, semitree, and their duals, which are satisfied by $T_{1}$-spaces, resemble separation axioms, while upward directed and downward directed are more like connectedness in that they restrict the number of open sets. In fact, the third characterization of an upward directed topology is Levine's definition of "strongly connected" [10], which is even stronger than $\boldsymbol{R}$-connected in that all upward directed sets (and all downward directed sets) are $R$-connected. Upper and lower semilattices restrict the number of open sets, but are also always $T_{0}$, so that they represent an intermediate band of topologies between $T_{0}$ and $T_{1}$.

Finally we note that if $T_{p}$ is an order-induced topological property with corresponding order property $K_{p}$, then $T_{p}$ is hereditary iff $K_{p}$ is hereditary, and $T_{p}$ is productive iff $K_{p}$ is productive. Furthermore, $K_{p}$ is hereditary (productive) iff the dual of $K_{p}$ is hereditary (productive). Using this and some appropriate examples [see 22, p. 65-68] it is not difficult to see that properties $T_{1}, T_{0}$, nested, $R_{0}, T_{F}, T(\beta), T(\beta, 0), T_{F F}, T(\epsilon), T_{Y}, T_{F C}$, and semiroot, as well as their duals, are all hereditary, while $T_{0}$-axiome multiplicatif, $R$-connected, $T(\gamma), T(\zeta), T(\delta)$, upward directed, and lower semilattice are not. Similarly, $T_{1}, T_{0}, T_{0}$-axiome multiplicatif, $R_{0}, T(\zeta)$, upward directed, upper semilattice, and their duals are productive, while the other properties are not.

3. Maximal and minimal topologies. In Theorems 3.1 through 3.6 we develop a general method for characterizing maximal and minimal $T_{p}$ when $T_{p}$ is order-induced, and, in Theorems 3.7 and 3.8, we describe maximal $T_{p}$ or minimal $T_{p}$ for most of the specific properties discussed in part 2. The conditions in Theorem 3.1 for maximal $T_{p}$ apply to all order-induced properties, while the corresponding conditions for minimal $T_{p}$ in Theorem 3.6 hold for most properties under discussion, but not all. In fact, Example 3.11 illustrates that for $T\left(\delta^{\prime}\right)$ and for the class of semitree topologies, the conclusion of Theorem 3.6 does not hold.

THEOREM 3.1. Let $T_{p}$ be an order-induced topological property and 
let $K_{p}$ be the corresponding order property. Let $\mathscr{T}$ be a topology on $X$ with preorder $R=\rho(\mathscr{T})$. $\mathscr{T}$ is a maximal $T_{p}$-topology iff $R$ is a minimal $K_{p}$-relation and $\mathscr{T}$ is the kernel topology $\nu(R)$.

Proof. Assume that $\mathscr{T}=\nu(R)$ and that $R$ is a minimal $K_{p}$ relation. Let $\mathscr{T}^{*} \in T_{p}$ such that $\mathscr{T} \subseteq \mathscr{T}^{*}$. Then $R=\rho(\mathscr{T}) \supseteq$ $\rho\left(\mathscr{T}^{*}\right) \in K_{p} \Rightarrow R=\rho\left(\mathscr{T}^{*}\right)$, since $R$ is minimal $K_{p}$. Therefore, by Theorem 1.1, $\mathscr{T}^{*} \subseteq \nu(R)=\mathscr{T} \Rightarrow \mathscr{T}^{*}=\mathscr{T}$, and $\mathscr{T}$ is maximal $T_{p}$.

Conversely, assume $\mathscr{T}$ is a maximal $T_{p}$-topology. Then $\mathscr{T} \subseteq$ $\nu(R) \in T_{p} \Rightarrow \mathscr{T}=\nu(R)$. Let $R^{*} \in K_{p}$ such that $R \supseteq R^{*}$. Then, since $\nu$ reverses order, $\mathscr{T}=\nu(R) \subseteq \nu\left(R^{*}\right) \in T_{p} \Rightarrow \nu(R)=\nu\left(R^{*}\right)$, because $\mathscr{T}$ is maximal. Therefore, $R=R^{*}$, and $R$ is a minimal $K_{p}$-relation.

The following can be proved in a similar manner.

Theorem 3.2. Let $T_{p}, K_{p}, X, \mathscr{T}$, and $R$ be as in Theorem 3.1.

(a) If $R$ is a maximal $K_{p}$-relation and $\mathscr{T}$ is the point-closure topology $\mu(R)$, then $\mathscr{T}$ is a minimal $T_{p}$-topology on $X$.

(b) If $\mathscr{T}$ is a minimal $T_{p}$-topology on $X$, then $\mathscr{T}=\mu(R)$.

Unfortunately, it is not necessary that $R_{\mathscr{g}}$ be maximal $K_{p}$ in order for $\mathscr{T}$ to be minimal $T_{p}$, as Example 3.11 illustrates. The difficulty is that $\mu$, unlike the function $\nu$, is not order-reversing, as was shown in Example 1.5. One additional restriction on the class $K_{p}$ which does make it necessary for $R_{\mathscr{T}}$ to be maximal is to assume that, whenever $R$ is a non-maximal $K_{p}$-relation, there is a "right finite" relation $S$ such that $R \subsetneq R \vee S \in K_{p}$. Before proving the Theorem, however, we derive three lemmas which develop a technique for creating coarser topologies by intersecting a given topology with a finite number of principal ultratopologies. The importance of these lemmas is that, in general, if $\mathscr{T}=\mathscr{T}_{1} \cap \mathscr{T}_{2}$, we can conclude only that $\rho(\mathscr{T}) \supseteq \rho\left(\mathscr{T}_{1}\right) \vee \rho\left(\mathscr{T}_{2}\right)$. But, whenever $\mathscr{T}_{2}$ is the intersection of a finite number of principal ultratopologies (that is, $\mathscr{T}_{2}=\nu(S)$ where $S$ is right-finite), then $\rho(\mathscr{T})=$ $\rho\left(\mathscr{T}_{1}\right) \vee \rho\left(\mathscr{T}_{2}\right)$.

Lemma 3.3. Let $R$ be any preorder on $X$, let $A \subseteq X$ and $b \in X$, let $S=\{\langle x, b\rangle: x \in A\} \cup \Delta$, and let $A_{b}=\{x:\langle b, x\rangle \notin R\}$. Then $S$ is a partial order and

$$
R \vee S=R \cup\{\langle x, y\rangle: x R a \text { and } b R y \text { for some } a \in A\}
$$

If $A=A_{b}$, then $R \vee S=R \cup\left\{\langle x, y\rangle: x \in A_{b}\right.$ and $\left.b R y\right\}$. Furthermore, if $A \subseteq A_{b}$ and $R$ is a partial order, then $R \vee S$ is a partial order. (The dual of this lemma is also valid.) 
Proof. It is clear that $S$ is a partial order and that $R \vee S \supseteq$ $R \cup\{\langle x, y\rangle: x R a$ and $b R y$ for some $a \in A\}$. Let $\langle x, y\rangle \in R \vee S$ such that $\langle x, y\rangle \notin R$. Then there is a finite sequence $x=x_{0}, \cdots, x_{n}=y$ such that $\left\langle x_{i-1}, x_{i}\right\rangle \in R \cup S, i=1, \cdots, n$, and this sequence can be chosen to be of minimal length. All elements of this sequence are then distinct and it is clear that $S$ can occur only once and $R$ cannot occur twice in a row, because, otherwise, the sequence could be shortened. Thus there are only four possibilities: $x=a S b=y, \quad x R a S b=y, \quad x=a S b R y$, and $x R a S b R y$, where $a$ is some element of $A$. In each case, $\langle x, y\rangle$ is such that $x R a$ and $b R y$ for some $a \in A$. Therefore, $R \vee S=$ $R \cup\{\langle x, y\rangle: x R a$ and $b R y$ for some $a \in A\}$.

Now assume that $A=A_{b}$. Let $\langle x, y\rangle$ be such that $x R a$ and $b R y$ for some $a \in A_{b}$. Since $\langle b, x\rangle \in R \Rightarrow\langle b, a\rangle \in R$, which is impossible for $a \in A_{b}$, we have $\langle b, x\rangle \notin R$, and thus $x \in A_{b}$. That is,

$\left\{\langle x, y\rangle: x R a\right.$ and $b R y$ for some $\left.a \in A_{b}\right\} \subseteq\left\{\langle x, y\rangle: x \in A_{b}\right.$ and $\left.b R y\right\}$.

Since containment in the other direction is immediate, the two sets are equal, and thus $R \vee S=R \cup\left\{\langle x, y\rangle: x \in A_{b}\right.$ and $\left.b R y\right\}$.

Finally, for $A \subseteq A_{b}$, if $\langle x, y\rangle \in R \vee S$, and $\langle y, x\rangle \in R \vee S$, then $\langle x, y\rangle$ and $\langle y, x\rangle$ are both in $R$. Otherwise, if at least one is not in $R$, it is easy to verify that $\langle b, a\rangle \in R$ for some $a \in A_{b}$, which is a contradiction. Therefore, if $R$ is antisymmetric, so also is $R \vee S$.

Lemma 3.4. Let $b \in X, A \subseteq X, S=\{\langle x, b\rangle: x \in A\} \cup \Delta$, and $\mathscr{T}_{2}=$ $\nu(S)$. If $\mathscr{T}=\mathscr{T}_{1} \cap \mathscr{T}_{2}$ where $\mathscr{T}_{1}$ is any topology on $X$, then $\rho(\mathscr{T})=$ $\rho\left(\mathscr{T}_{1}\right) \vee \rho\left(\mathscr{T}_{2}\right)$

Proof. Let $R=\rho\left(\mathscr{T}_{1}\right) . \quad \mathscr{T} \subseteq \mathscr{T}_{1} \Rightarrow \rho(\mathscr{T}) \supseteq \rho\left(\mathscr{T}_{1}\right)=R$, and $\mathscr{T} \subseteq$ $\mathscr{T}_{2} \Rightarrow \rho(\mathscr{T}) \supseteq \rho\left(\mathscr{T}_{2}\right)=S$. Therefore, $\rho(\mathscr{T}) \supseteq R \vee S$. It remains to show that $\rho(\mathscr{T}) \subseteq R \vee S$.

Let $p, q \in X$ such that $\langle p, q\rangle \notin R \vee S$. To show $\langle p, q\rangle \notin \rho(\mathscr{T})$, it suffices to find a neighborhood $N$ of $q$ in $\mathscr{T}=\mathscr{T}_{1} \cap \mathscr{T}_{2}$ such that

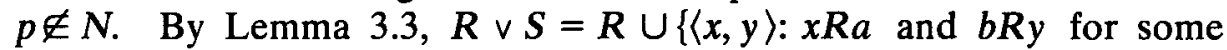
$a \in A\}$. Therefore, $\langle p, q\rangle \notin R$ and there is no $a \in A$ such that $p R a$ and $b R q$. Since $\langle p, q\rangle \notin R$, there is an open neighborhood $N_{1}$ of $q$ in $\mathscr{T}_{1}$ with $p \notin N_{1}$. By Theorem 1.4, $\mathscr{T}_{2}=\nu(S)=\cap\{\mathscr{T}(y, x):\langle x, y\rangle \in S\}=$ $\cap\{\mathscr{T}(b, x): x \in A\}$.

Case $\langle b, q\rangle \notin R$. There is an open neighborhood $N_{2}$ of $q$ in $\mathscr{T}_{1}$ with $b \notin N_{2}$. Then $N \equiv N_{1} \cap N_{2}$ is an open neighborhood of $q$ in $\mathscr{T}_{1}$ containing neither $p$ nor $b$. $b \notin N \Rightarrow N \in \mathscr{P}(\sim\{b\}) \subseteq \mathscr{T}(b, x) \forall x \in A$ $\Rightarrow N \in \mathscr{T}_{2} \Rightarrow N \in \mathscr{T}_{1} \cap \mathscr{T}_{2}=\mathscr{T}$. Therefore, $N$ is an open neighborhood of $q$ in $\mathscr{T}$ excluding $p$, and $\langle p, q\rangle \notin \rho(\mathscr{T})$.

Case $\langle b, q\rangle \in R$. Then for each $x \in A,\langle p, x\rangle \notin R$, and there is an 
open neighborhood $N_{x}$ of $x$ in $\mathscr{T}_{1}$ excluding $p$. Let $N=$ $N_{1} \cup\left(\cup\left\{N_{x}: x \in A\right\}\right)$, which is open in $\mathscr{T}_{1}$ and still excludes $p$. For each $x \in A, \quad x \in N \Rightarrow N \in \mathscr{U}(x) \subseteq \mathscr{T}(b, x)$. Therefore, $\quad N \in \cap\{\mathscr{T}(b, x)$ : $x \in A\}=\mathscr{T}_{2} \Rightarrow N \in \mathscr{T}$. Since $q \in N \in \mathscr{T}$ and $p \notin N,\langle p, q\rangle \notin \rho(\mathscr{T})$.

In both cases, $\langle p, q\rangle \notin \rho(\mathscr{T})$, and thus $\rho(\mathscr{T}) \subseteq R \vee S$. Therefore, $\rho(\mathscr{T})=R \vee S=\rho\left(\mathscr{T}_{1}\right) \vee \rho\left(\mathscr{T}_{2}\right)$.

Definition. A relation $R$ on $X$ is right-finite iff $\{y:\langle x, y\rangle \in R \sim \Delta$ for some $x \in X\}$ is finite. $R$ is finite iff $R \sim \Delta$ is a finite set.

LEMMA 3.5. Let $S$ be a right-finite preorder relation on $X$ and let $\mathscr{T}_{2}=\nu(S)$. If $\mathscr{T}=\mathscr{T}_{1} \cap \mathscr{T}_{2}$ where $\mathscr{T}_{1}$ is any topology on $X$, then $\rho(\mathscr{T})=$ $\rho\left(\mathscr{T}_{1}\right) \vee \rho\left(\mathscr{T}_{2}\right)$.

Proof. Let $B=\{y:\langle x, y\rangle \in S$ for some $x \in X\}$, which is finite by hypothesis. For each $b \in B$, let $S_{b}=\{\langle x, b\rangle:\langle x, b\rangle \in S\} \cup \Delta$. Then $S=$ $\cup\left\{S_{b}: b \in B\right\}=\vee\left\{S_{b}: b \in B\right\}$. Since each $S_{b}$ is of the form of the relation $S$ in Lemma 3.4, the proof follows by induction on the cardinality of $B$, using Lemma 3.4 and the associativity of $\cap$ for topologies and of $v$ for relations.

THEOREM 3.6. Let $T_{p}$ be an order-induced topological property and let $K_{p}$ be the corresponding order property. Assume that, whenever $R \in K_{p}$ and $R$ is not a maximal $K_{p}$-relation, there is a right-finite preorder $S$ such that $R \subsetneq R \vee S \in K_{p}$. Let $\mathscr{T}$ be a topology on $X$. Then $\mathscr{T}$ is a minimal $T_{p}$-topology iff $R_{\mathscr{G}}$ is a maximal $K_{p}$-relation and $\mathscr{T}$ is the point-closure topology $\mu\left(R_{\mathscr{F}}\right)$.

Proof. That this condition is sufficient was proved in Theorem 3.2. To show necessity, let $\mathscr{T}$ be a minimal $T_{p}$-topology and let $R=R_{\mathscr{g}}$. Then, by Theorem 3.2, $\mathscr{T}=\mu(R)$. Suppose $R$ is not a maximal $K_{p}$-relation. Then there is a right-finite relation $S$ on $X$ such that $R \subsetneq R \vee S \in K_{p}$. Let $\mathscr{T}^{*}=\mathscr{T} \cap \nu(S)$. By Lemma 3.5, $\rho\left(\mathscr{T}^{*}\right)=$ $\rho(\mathscr{T}) \vee \rho(\nu(S))=R \vee S \in K_{p}$, and thus $\mathscr{T}^{*} \in T_{p}$. If $\mathscr{T}^{*}=\mathscr{T}$, then $\rho\left(\mathscr{T}^{*}\right)=\rho(\mathscr{T}) \Rightarrow R \vee S=R$, which is a contradiction. Therefore, $\mathscr{T}^{*} \subsetneq \mathscr{T}$ and $\mathscr{T}^{*} \in T_{p}$, which violates the $T_{p}$-minimality of $\mathscr{T}$. Thus $R$ is a maximal $K_{p}$-relation.

We now apply Theorems 3.1 and 3.6 to characterize maximal $T_{p}$ or minimal $T_{p}$ for most of the properties discussed in part 2. Properties such as nested, upward directed, upper-semilattice, and $R$-connected tend to restrict the number of open sets and to have nontrivial maximal spaces. Most of the other properties, however, by restricting the elements in the relation and increasing the number of open sets, are more like separation axioms and have nontrivial minimal spaces. 
Determinimg maximal $T_{p}$ or minimal $T_{p}$ is, in most cases, primarily a matter of characterizing minimal $K_{p}$ or maximal $K_{p}$. These characterizations are usually intuitively obvious from consideration of appropriate Hasse diagrams, even though formal verification may be tedious or even complicated. For this reason, a few portions of proofs will be included as illustrations, and the rest will be omitted.

THEOREM 3.7. Let $\mathscr{T}$ be a topology on $X$ and let $R=\rho(\mathscr{T})$. $\nu(R)$.

(a) $\mathscr{T}$ is maximal nested iff $R$ is linear and $\mathscr{T}$ is the kernel topology,

(b) $\mathscr{T}$ is a maximal upward directed (strongly connected) topology iff $R$ is a root of length 1 and $\mathscr{T}=\nu(R)$.

(c) $\mathscr{T}$ is a maximal upper semilattice topology iff $R$ is a root of length 1 and $\mathscr{T}=\nu(R)$.

(d) $(X, \mathscr{T})$ is a maximal $R$-connected space iff $\mathscr{T}=\nu(R)$, and, for all $x \neq y \in X$, there is a unique finite sequence $x=x_{0}, \cdots, x_{n}=y$ of distinct points connecting $x$ to $y$ in $R$.

Proof. By Theorem 3.1, it suffices to characterize minimal $K_{p}$ relations for each of the four properties. For example in (a) it suffices to show that $R$ is a minimal totally ordered preorder iff $R$ is linear. In each case, it is easy to verify the sufficiency of the condition for $R$ to be minimal $K_{p}$, and we restrict our attention to an outline of the proof of necessity.

(a) Let $R$ be a totally ordered preorder and suppose $R$ is not linear. Then $\exists a \neq b$ with $\langle a, b\rangle \in R$ and $\langle b, a\rangle \in R$. Let $R^{*} \equiv$ $R \sim\{\langle x, b\rangle: a R x R b$ and $x \neq b\}$. It can be verified that $R^{*}$ is also a totally ordered preorder and, since $\langle a, b\rangle \notin R^{*}, R^{*} \subsetneq R$. Therefore, $R$ is not minimal.

(b) Let $R$ be a minimal upward directed relation. If $R$ is not a partial order, then $\exists a \neq b$ with $\langle a, b\rangle \in R$ and $\langle b, a\rangle \in R$. As in part (a), $R^{*} \equiv R \sim\{\langle x, b\rangle: a R x R b$ and $x \neq b\}$ is a preorder such that $R^{*} \subsetneq R$, and it is easy to show that $R^{*}$ is also upward directed. This is a contradiction, and $R$ is therefore a partial order. If $R$ has a chain, $a<b<c$, of length 2 , then, as before, $R^{*} \equiv R \sim\{\langle x, b\rangle: a R x R b$ and $x \neq b\}$ is an upward directed preorder such that $R * \subsetneq R$. Therefore, $R$ has length at most 1. Finally, since $|X| \geqq 2$ and $R$ is upward directed, there is an $\langle a, b\rangle \in R \sim \Delta$. If $x$ is any other element of $X$, then $x$ and $b$ have an upper bound $u$; and, since $a<b \leqq u$ and $R$ has length $1, u=b$. That is, $x \leqq b$ for all $x \in X$ and $b$ is a greatest element. Therefore, $R$ is a root of length 1 .

(c) Let $R$ be a minimal upper semilattice. If $R$ has a chain, $a<b<c$, of length 2 , then it can be verified that $R^{*} \equiv$ $R \sim\{\langle x, y\rangle \in R: x \neq y$ and $\langle c, y\rangle \notin R\}$ is an upper semilattice and that 
$R * \subsetneq R$. Therefore, $R$ is a partial order of length 1 . That $R$ has a greatest element then follows as in part (b), and $R$ is a root of length 1 .

(d) Let $R$ be a minimal connected relation. Let $c, d \in X$ and let $c=a_{0}, \cdots, a_{n}=d$ be a sequence of shortest length connecting $c$ to $d$. Then $\left\langle a_{t}\right\rangle$ is a sequence of distinct points. Suppose $c=b_{0}, \cdots, b_{m}=$ $d$ is any other sequence of distinct points connecting $c$ to $d$. Then $n \leqq m$. Let $p$ be the first $i$ such that $a_{1} \neq b_{v}$, let $k$ be the first $i>p$ such that $b_{l}=a_{1}$ for some $j$, and let $q$ be that integer such that $b_{k}=a_{q}$. Then $0<p<k, p \leqq q$, and $\left\langle a_{p-1}, b_{p}\right\rangle=\left\langle b_{p-1}, b_{p}\right\rangle \in R \cup R^{-1}$. If $\left\langle a_{p-1}, b_{p}\right\rangle \in R$, let $a=a_{p-1}$ and $b=b_{p}$. If $\left\langle b_{p}, a_{p-1}\right\rangle \in R$, let $a=b_{p}$ and $b=a_{p-1}$. In either case, as in part (a), $R^{*} \equiv R \sim\{\langle x, b\rangle: a R x R b$ and $x \neq b\}$ is a preorder such that $R^{*} \mp R$, and it can be verified that $R^{*}$ is connected. Therefore, there is no such other sequence $b_{0}, \cdots, b_{m}$, and the original sequence $a_{0}, \cdots, a_{n}$ is unique.

Since $\mathscr{T}=\nu(\rho(\mathscr{T}))$ iff $\mathscr{T}$ is principal, it is possible to rephrase the characterizations in Theorem 3.7 in more topological terms by using the correspondences in part 2 , as follows. $\mathscr{T}$ is maximal nested iff $\mathscr{T}$ is a principal $T_{0}$ nested topology. $\mathscr{T}$ is maximal upward directed (or a maximal upper semilattice topology) iff $\mathscr{T}$ is a principal connected $T(\beta, 0)$-topology. $(X, \mathscr{T})$ is a maximal $R$-connected space iff $(X, \mathscr{T})$ is a principal $T_{Y}$-space and for all $x, y \in X$ there is a unique simple chain of kernels from $x$ to $y$.

This latter characterization of maximal $R$-connected requires some explanation. Proof of necessity is straightforward although rather lengthy. One way to show sufficiency is to note that this condition is equivalent to one given by Thomas [17] to characterize maximal connected for principal topological spaces. Then, since it is easy to show that principal maximal connected $\Rightarrow$ maximal principal connected $\Rightarrow$ maximal $R$-connected, sufficiency follows. Consequently, the maximal $R$-connected spaces are precisely the principal maximal connected spaces.

If $K_{p}$ and $K_{p^{\prime}}$ are dual order properties, then minimal $K_{p^{\prime}}$ is the dual of minimal $K_{p}$ and maximal $K_{p}$ is the dual of maximal $K_{p}$. Therefore, using Theorem 3.1, the characterization of maximal $T_{p^{\prime}}$ follows easily from that of maximal $T_{p}$. In particular, $\mathscr{T}$ is a maximal downward directed topology iff $R$ is a tree of length 1 and $\mathscr{T}=\nu(R)$, and the same for maximal lower semilattice topologies. (The other two properties in Theorem 3.7 are self-dual.)

Note, however, that, even though $T_{p}$ and $T_{p^{\prime}}$ are dual order-induced properties, maximal $T_{p}$ and maximal $T_{p^{\prime}}$ are usually not dual properties, and neither are minimal $T_{p}$ and minimal $T_{p^{\prime}}$. In fact, maximal $T_{p}$ cannot even be order-induced unless all minimal $K_{p}$-relations $R$ are such that $\mu(R)=\nu(R)$. Similarly, minimal $T_{p}$ is order-induced iff $\mu(R)=\nu(R)$ for all maximal $K_{p}$-relations. Of the minimal and maximal properties 
characterized in this paper, only minimal $T(\zeta)$ and minimal $T(\delta)$ are order-induced.

To characterize minimal $T_{p}$-topologies by Theorem 3.6 one must do two things: (1) characterize maximal $K_{p}$ and (2) show that whenever $R$ is a nonmaximal $K_{p}$-relation there is a right-finite preorder $S$ such that $R \subsetneq R \vee S \in K_{p}$. In Theorem 3.8, the inherent characterizations of maximal $K_{p}$ are intuitively obvious in each case, and in parts (b)-(g) the actual verifications of (1) and (2) are completely straightforward. ((a) is a trivial application of Theorem 1.1.) Proofs of the last three parts are slightly more involved, and (i) and (h) will be considered as examples. It is possible in most cases to choose the relation $S$ of the form $\{\langle a, b\rangle\} \cup \Delta$ which is actually finite, but for $K(\delta)$, semiroots, and semilattices, however, $S$ is of the form $\left\{\langle x, b\rangle: x \in A_{b}\right\} \cup$ $\Delta$, which is right-finite, but not necessarily finite.

THEOREM 3.8. Let $\mathscr{T}$ be a topology on $X$ and let $R=\rho(\mathscr{T})$.

(a) The minimum $T_{1}$-topology on $X$ is $\mu(\Delta)$.

(b) $\mathscr{T}$ is a minimal $T_{0}$-topology iff $R$ is linear and $\mathscr{T}$ is the pointclosure topology, $\mu(R)$.

(c) $\mathscr{T}$ is a minimal $T_{F}$-topology iff $\mathscr{T}=\mu(R)$ and $R$ is a partial order of length 1 such that every maximal element is greater than every minimal element.

(d) $\mathscr{T}$ is a minimal $T(\beta)$-topology iff $\mathscr{T}=\mu(R)$ and $R$ is a presemiroot of length 1 such that, if $R$ has a singleton $R$-component, then $R$ is an equivalence relation and every other component has exactly two elements.

(e) $\mathscr{T}$ is a minimal $T(\beta, 0)$-topology iff $\mathscr{T}=\mu(R)$ and all $R$ components are roots of length 1 (or, equivalently, iff $\mathscr{T}=\mu(R)$ and $R$ is a semiroot of length 1 with no singleton components).

(f) $\mathscr{T}$ is a minimal $T_{F F}$-topology iff $\mathscr{T}=\mu(R)$ and $(X, R)$ is a root or tree of length 1.

(g) If $X$ is an infinite set, there are no minimal $T_{F C}$-topologies on $X$.

(h) $\mathscr{T}$ is a minimal $T(\zeta)$-topology iff $R$ is a linear order such that every nonmaximum element has a successor.

(i) $\mathscr{T}$ is a minimal $T(\delta)$-topology iff $R$ is a linear order such that every nonmaximum element has a successor.

(j) $\mathscr{T}$ is a minimal semiroot-topology iff $\mathscr{T}=\mu(R)$ and $R$ is a linear order. Minimal semi-lattice topologies and minimal $T_{0}$-upward directed topologies have the same characterization.

Proof of (i). We first show that, whenever $R$ is a nonlinear $K(\delta)$-relation, there is a right-finite preorder $S$ such that $R \vee S$ is a $K(\delta)$-relation and $R \subsetneq R \vee S$. Let $R$ be a nonlinear $K(\delta)$ - 
relation. Then $\exists a, b \in X$ such that $\langle a, b\rangle \notin R$ and $\langle b, a\rangle \notin R$. Let $A_{b}=\{x:\langle b, x\rangle \notin R\}$ and let $S=\left\{\langle x, b\rangle: x \in A_{b}\right\} \cup \Delta$. Then $S$ is a rightfinite preorder, and, by Lemma $3.3, R^{*} \equiv R \vee S$ is a partial order such that $\quad R \subsetneq R^{*}$. Furthermore, $R \vee S=R \cup\left\{\langle x, y\rangle: x \in A_{b} \quad\right.$ and $b R y\}$. Let $p$ be a nonmaximal element of $\left(X, R^{*}\right)$. If $p$ is maximal in $(X, R)$, then $p \neq b$, and $b$ is a successor for $p$ in $R^{*}$. Assume $p$ is nonmaximal in $(X, R)$. Then $p$ has a successor, $q$, in $R$. If $p \notin A_{b}$, then $q$ is still a successor for $p$ in $R^{*}$. If $p \in A_{b}$, then $q^{*} \equiv \min _{R^{*}}\{q, b\}$ is a successor for $p$ in $R^{*}$. Thus, in all cases, $p$ has a successor in $R^{*}$, and $R^{*} \in K(\delta)$. Therefore, there is a right-finite relation $S$ such that $R \subsetneq R \vee S \in K(\delta)$.

The preceding construction also shows that a nonlinear $K(\delta)$ relation cannot be maximal $K(\delta)$. Conversely, a linear $K(\delta)$-relation is clearly maximal $K(\delta)$, because any relation properly containing $R$ is not a partial order and thus not $K(\delta)$. Therefore, $R$ is a maximal $K(\delta)$ relation iff $R$ is a linear order such that every nonmaximum element has a successor. Furthermore, if $R$ is a nonmaximal $K(\delta)$-relation, then $R$ is nonlinear, and there is a right-finite relation $S$ such that $R \subsetneq R \vee S \in$ $K(\delta)$. Therefore, by Theorem 3.6, $\mathscr{T}$ is minimal $T(\delta)$ iff $\mathscr{T}=\mu(R)$ and $\boldsymbol{R}$ is a linear order such that every nonmaximum element has a successor. But by Theorem 1.3, under these conditions on $R, \mu(R)=$ $\nu(R)$, and $\mu(R)$ is the only topology with relation $R$, so that (i) follows.

Proof of (h). We show only that, whenever $R$ is a nonlinear $K(\zeta)$-relation, there is a finite preorder $S$ such that $R \subsetneq R \vee S \in$ $K(\zeta)$. The remainder of the proof then follows as for minimal $T(\delta)$ topologies in (i), with the observation that, for a linear order, a cover is the same as a successor.

Let $R$ be a nonlinear $K(\zeta)$-relation. Then $\exists a, b \in X$ such that $\langle a, b\rangle \notin R$ and $\langle b, a\rangle \notin R$. Let $S=\{\langle a, b\rangle\} \cup \Delta$ and let $R^{*}=$ $R \vee S$. Then $S$ is a finite preorder, and, by Lemma $3.3, R^{*}=$ $R \cup\{\langle x, y\rangle: x R a$ and $b R y\}$ is a partial order such that $R \subsetneq R^{*}$. It is routine to verify that $b$ covers $a$ in $R^{*}$ and also that, if $c$ covers $s$ in $R$ and $\langle s, a\rangle \notin R$ or $\langle b, c\rangle \notin R$, then $c$ covers $s$ in $R^{*}$.

Let $s, t \in X$ such that $s<t$ in $R^{*}$. It suffices to show the existence of a cover $c$ of $s$ in $R^{*}$ such that $\langle c, t\rangle \in R^{*}$. In the case that $\langle s, t\rangle \notin R$, then $s R a S b R t$. If $s=a$, then $b$ covers $a$ and $\langle b, t\rangle \in R^{*}$. If $s \neq a$, then $s$ has a cover $c$ in $R$ and $c \leqq a$ in $R$. $\langle c, a\rangle \in R$ and $\langle b, a\rangle \notin R \Rightarrow$ $\langle b, c\rangle \notin R$. Therefore, $c$ covers $s$ in $R^{*}$ and $\langle c, t\rangle \in R^{*}$. Now assume $\langle s, t\rangle \in R$. Then $s$ has a cover $c$ in $R$ with $\langle c, t\rangle \in R$. If $\langle s, a\rangle \notin R$ or $\langle b, c\rangle \notin R$, then $c$ covers $s$ in $R^{*}$. Suppose $s R a$ and $b R c$. If $s=a$, then $b$ covers $a$ and $\langle b, t\rangle \in R^{*}$. If $s \neq a$, then $s$ has a cover $c_{1}$ such that $c_{1} R a$. Then $\left\langle c_{1}, t\right\rangle \in R^{*}$, and, since $\left\langle b, c_{1}\right\rangle \notin R, c_{1}$ is still a cover of $s$ in $R^{*}$. Thus, in all cases, $s$ has a cover $c$ in $R^{*}$ such that $\langle c, t\rangle \in$ 
$R^{*}$. That is, $R^{*}$ is a $K(\zeta)$-relation, and $S$ is a finite preorder such that $R \subsetneq R \vee S \in K(\zeta)$.

Characterizations of minimum $T_{1}$ and minimal $T_{0}$ are, of course, already well-known. The statement in (b) is close to the theorem of Larson [9] and Pahk [15] that a $T_{0}$-topological space $(X, \mathscr{T})$ is minimal $T_{0}$ iff $\{\sim\{\bar{x}\}: x \in X\} \cup\{X\}$ is a base for $\mathscr{T}$ and finite unions of point closures are point closures.

Larson [9] has also proved that a $T_{D}$-topological space is minimal $T_{D}$ iff the topology is nested. Using the fact that when $\boldsymbol{R}_{\mathscr{F}}$ is linear the kernels are complements of derived sets, it is not difficult to prove as a corollary that a topological space $(X, \mathscr{T})$ is minimal $T_{D}$ iff $R_{\mathscr{F}}$ is linear and $\mathscr{T}$ is the kernel topology of $\boldsymbol{R}_{\mathscr{g}}$. Theorem 3.9 then follows immediately from Theorem 3.7.

THEOREM 3.9. A topological space $(X, \mathscr{T})$ is minimal $T_{D}$ iff it is maximal nested.

It is clear that a minimal $T_{D}$-topology with relation $R$ is also minimal $T_{0}$ precisely when $\mu(R)=\nu(R)$, that is, whenever $R$ is a linear order such that every nonmaximum element has a successor. This is also the condition for minimal $T(\zeta)$ and minimal $T(\delta)$.

Characterization of minimal $T_{p^{\prime}}$ does not follow as readily from minimal $T_{p}$ as did maximal $T_{p^{\prime}}$ from maximal $T_{p}$. The problem is in finding an appropriate right-finite relation $S$ in order to apply Theorem 3.6. In the case of $T(\beta), T(\beta, 0), T(\zeta)$, and upward directed $T_{0^{-}}$ topologies, if $R$ is nonmaximal $K_{p}$, there is a finite preorder $S=$ $\{\langle a, b\rangle\} \cup \Delta$ such that $R \subsetneq R \vee S \in K_{p}$. Then, by duality, $K_{p}$, also satisfies the hypothesis of Theorem 3.6, and Theorem 3.10 follows. $\left(T_{0}, T_{F}, T_{F F}\right.$, and $T_{F C}$ are self-dual.)

THEOREM 3.10. Let $\mathscr{T}$ be a topology on $X$ and let $R=\rho(\mathscr{T})$.

(a) $\mathscr{T}$ is minimal $T\left(\beta^{\prime}\right)$ iff $\mathscr{T}=\mu(R)$ and $R$ is a presemiroot of length 1 such that, if $R$ has a singleton $R$-component, then $R$ is an equivalence relation and every other component has exactly two elements.

(b) $\mathscr{T}$ is minimal $T_{Y S}$ (that is, minimal $T\left(\beta^{\prime}, 0\right)$ ) iff $\mathscr{T}=\mu(R)$ and all $R$-components are trees of length 1 .

(c) $\mathscr{T}$ is a minimal $T\left(\zeta^{\prime}\right)$-topology iff $\mathscr{T}=\mu(R)$ and $R$ is a linear order such that every nonminimum element has a predecessor.

(d) $\mathscr{T}$ is a minimal downward directed $T_{0}$-topology iff $\mathscr{T}=\mu(R)$ and $R$ is linear.

For $T(\delta)$, semiroot, and upper semilattice topologies, the crucial preorder in the proof of Theorem 3.8 is of the form $S=$ $\left\{\langle x, b\rangle: x \in A_{b}\right\} \cup \Delta$, which need not be finite. In the dual situation, the 
corresponding preorder is left-finite, but not necessarily right-finite, so that Theorem 3.6 does not apply, but only the rather unsatisfactory Theorem 3.2 giving sufficient conditions. That is, let $\mathscr{T}$ be a topology on $X$ and let $R=\rho(\mathscr{T})$. If $\mathscr{T}=\mu(R)$ and $R$ is linear, then $\mathscr{T}$ is a minimal semitree topology and a minimal lower semilattice topology. If, in addition, $R$ is such that every nonminimum element has a predecessor, then $\mathscr{T}$ is minimal $T\left(\delta^{\prime}\right)$. For minimal $T\left(\delta^{\prime}\right)$ and minimal semitree topologies, however, $R$ necd not be maximal $K_{p}$, as is shown by Example 3.11, below. (The question for minimal lower semilattice topologies has not been settled.)

EXAMPLE 3.11. Let $\omega$ be the first infinite ordinal and let $\omega_{1}$ be the first uncountable ordinal. Let $X_{1}$ be the underlying set for $\omega_{1}$, let $X_{0}$ be a relabeling of the elements of $\omega$ so that $X_{0} \cap X_{1}=\varnothing$, and let $X=$ $X_{0} \cup X_{1}$. Let $R_{0}$ and $R_{1}$ be the inverses of the usual well-orderings of $X_{0}$ and $X_{1}$, and let $R=R_{0} \cup R_{1}$. (For the rest of the example, symbols such as $\leqq$ and $\{\bar{x}\}$ will always refer to the relation $R$.) With the usual ordering of the ordinal numbers, every ordinal has a successor, every subset has a least element, and $\alpha \in \beta$ iff $\alpha<\beta$. Therefore, $(X, R)$ is a partially ordered set in which every element has a predecessor, every subset of $X_{0}$ has a greatest element, every subset of $X_{1}$ has a greatest element, and $\alpha \in \beta$ iff $\alpha>\beta$.

Let $\mathscr{T}=\mu(R)$ and let $\mathscr{C}$ be the closed sets of $\mathscr{T} . \quad \mathscr{T}$ is clearly a $T\left(\delta^{\prime}\right)$ semitree topology, and, since $R$ is nonlinear, $R$ is neither a maximal semitree nor a maximal $K\left(\delta^{\prime}\right)$-relation. We will show that $\mathscr{T}$ is nevertheless a minimal semitree topology and a minimal $T\left(\delta^{\prime}\right)$-topology.

$\mathscr{C}$ consists of $\varnothing, X$, and sets of the form $\{\bar{m}\}=\{n: m \leqq n\},\{\bar{x}\}=$ $\{y: x \leqq y\}$, and $\{\bar{m}\} \cup\{\bar{x}\}$, where $m \in X_{0}$ and $x \in X_{1}$. Sets of the form $X_{0} \cup\{\bar{x}\},\{\bar{m}\} \cup X_{1}$, and $\{\bar{m}\} \cup \beta$, where $x \in X_{1}, m \in X_{0}$, and $\beta$ is a limit ordinal in $\omega_{1}$, are not closed in $(X, \mathscr{T})$. (In terms of the inverse ordering $R$, a limit ordinal is an ordinal other than 0 with no successor.) It is on the fact that these sets are not closed that the proof is based.

(a) To show that $\mathscr{T}$ is a minimal semitree topology, suppose there is a semitree topology $\mathscr{T}^{*}$ on $X$ with closed sets $\mathscr{C}^{*}$ such that $\mathscr{T} \supsetneq \mathscr{T}^{*}$, and let $R^{*}=\rho\left(\mathscr{T}^{*}\right)$. Then $R \subseteq R^{*}$, and, since $\mathscr{T}$ is the smallest topology with relation $R, R \subsetneq R^{*}$. Therefore, since $R^{*}$ is a partial order, there are elements $p \in X_{0}$ and $a \in X_{1}$ such that $p$ and $a$ are related in $R^{*}$. Since $R^{*}$ is a semitree, $\{\hat{p}\}^{*}$ and $\{\hat{a}\}^{*}$ are both linear, and thus $n$ is $R^{*}$ related to $x$ for all $n \leqq p$ and $x \leqq a$.

Let $n$ be any element of $X_{0}$ such that $n \leqq p$. If $\langle n, x\rangle \in R^{*}$ for all $x \leqq a$, then $\{\bar{n}\} \cup X_{1}=\{\bar{n}\}^{*} \in \mathscr{C}^{*} \subseteq \mathscr{C}$, which is a contradiction, because 
such sets are not closed in $(X, \mathscr{T})$. Therefore, there is an element $\varphi(n) \leqq a$ in $X_{1}$ such that $\langle n, \varphi(n)\rangle \notin R^{*}$. That is, $\langle\varphi(n), n\rangle \in R^{*}$.

For each $n \leqq p, \quad \varphi(n) \in X_{1} \Rightarrow|\varphi(n)| \leqq|\omega|=\aleph_{0}$. Therefore, $|\cup\{\varphi(n): n \leqq p\}| \leqq \aleph_{0}$. Suppose there is no $t \in X_{1}$ such that $\varphi(n) \geqq t$ for all $n \leqq p$. Then, for each $x \in X_{1}, x>\varphi(n)$ and $x \in \varphi(n)$ for some $n \leqq p$. Therefore, $X_{1} \subseteq \cup\{\varphi(n): n \leqq p\}$ where the latter is a countable set. This is clearly impossible and thus there is a $t \in X_{1}$ such that $\varphi(n) \geqq t$ for all $n \leqq p$. Then $\langle t, n\rangle \in R^{*}$ for all $n \in X_{0}$, and $X_{0} \cup\{\bar{t}\}=$ $\{\bar{t}\}^{*} \in \mathscr{C}^{*} \subseteq \mathscr{C}$, which is impossible because $(X, \mathscr{T})$ has no such closed sets.

Thus there is no semitree topology $\mathscr{T}^{*}$ on $X$ such that $\mathscr{T} \supsetneq \mathscr{T}^{*}$, and $\mathscr{T}$ is a minimal semitree topology.

(b) To show that $\mathscr{T}$ is minimal $T\left(\delta^{\prime}\right)$, suppose there is a $T\left(\delta^{\prime}\right)$ topology $\mathscr{T}^{*}$ on $X$ such that $\mathscr{T} \supsetneqq \mathscr{T}^{*}$. Let $\mathscr{C}^{*}$ be the closed sets of $\left(X, \mathscr{T}^{*}\right)$ and let $R^{*}=\rho\left(\mathscr{T}^{*}\right) . \quad$ As in part (a), $R \subsetneq R^{*}$. Therefore, there are elements $v_{0} \in X_{0}$ and $v_{1} \in X_{1}$ such that $v_{0}$ and $v_{1}$ are related in $R^{*}$. We will eventually show the existence of $p \in X_{0}$ and $a \in X_{1}$ such that $\langle a, p\rangle \in R^{*}$ and $n$ is $R^{*}$ related to $x$ for all $n \leqq p$ and all $x \leqq a$.

We may assume that there exist $v_{0} \in X_{0}$ and $v_{1} \in X_{1}$ such that $\left\langle v_{0}, v_{1}\right\rangle \in R^{*}$. To show this, first assume that $\left\langle u_{1}, u_{0}\right\rangle \in R^{*}$ where $u_{0} \in$ $X_{0}$ and $u_{1} \in X_{1}$. Let $r$ be the greatest element of $\left(X_{1}, R_{1}\right)$ such that $\langle r, q\rangle \in R^{*}$ for some $q \in X_{0}$. There is an $m \in X_{0}$ such that $\langle r, m\rangle \notin R^{*}$, because, otherwise, $\langle r, n\rangle \in R^{*}$ for all $n \in X_{0}$ and $X_{0} \cup\{\bar{r}\}=\{\bar{r}\}^{*} \in \mathscr{C}^{*} \subseteq$ $\mathscr{C}$, which is impossible. Therefore, there is a greatest element $k$ of $X_{0}$ such that $\langle r, k\rangle \notin R^{*}$. Since $k<q, k$ has a successor $k^{+}$in $\left(X_{0}, R_{0}\right)$, and $\left\langle r, k^{+}\right\rangle \in R^{*}$. Then $k$ and $r$ are related (otherwise, $k^{+}$covers both $k$ and $r$ and $k^{+}$has no predecessor), and, since $\langle r, k\rangle \notin R^{*},\langle k, r\rangle \in R^{*}$. Thus there exist $v_{0} \in X_{0}$ and $v_{1} \in X_{1}$ such that $\left\langle v_{0}, v_{1}\right\rangle \in R^{*}$.

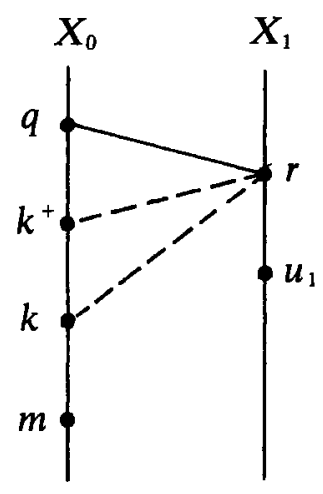

Let $p$ be the greatest element of $X_{0}$ such that $\langle p, s\rangle \in R^{*}$ for some $s \in X_{1}$. If $\langle p, x\rangle \in R^{*}$ for all $x \in X_{1}$, then $X_{1} \cup\{\bar{p}\}=\{\bar{p}\}^{*} \in \mathscr{C}^{*} \subseteq \mathscr{C}$, which is a contradiction. Therefore, there is a greatest element $a$ in $X_{1}$ such that $\langle p, a\rangle \notin R^{*}$. Then $\langle p, x\rangle \in R^{*}$ for all $x>a$, and 


$$
a \cup\{\bar{p}\}=\{x: a<x\} \cup\{n: p \leqq n\}=\left\{x:\langle p, x\rangle \in R^{*}\right\}=\{\bar{p}\}^{*} \in \mathscr{C}^{*} \subseteq \mathscr{C}
$$

Therefore, $a$ cannot be a limit ordinal in $\omega_{1}$, and, since $a \neq 0, a$ has a successor, $a^{+}$in $R$ and $\left\langle p, a^{+}\right\rangle \in R^{*}$. By definition of $p$, there can be no element of $X_{0}$ between $p$ and $a^{+}$in $R^{*}$. Therefore, if $p$ and $a$ are unrelated in $R^{*}, a^{+}$covers both $p$ and $a$ and has no predecessor in $R^{*}$, which is a contradiction. Thus, $\langle a, p\rangle \in R^{*}$. Furthermore, since $a R^{*} p R^{*} a^{+}, p$ is related to every element of $X_{1}$.

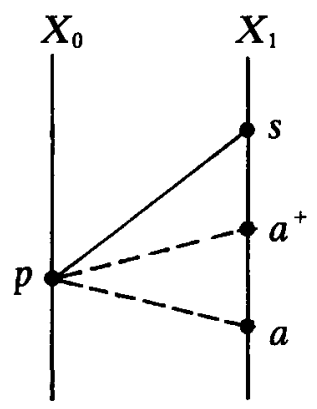

We next show by induction that each $n \leqq p$ is related by $R^{*}$ to each element of $X_{1}$. Assume that $k$ is related to each element of $X_{1}$, where $k \leqq p$. It suffices to show that $k^{-}$, the predecessor of $k$ in $R$, is related to every element of $X_{1}$. By the same argument as for $p$, it is impossible to have $\left\langle k^{-}, x\right\rangle \in R^{*}$ for all $x \in X_{1}$, and thus there is a greatest element $b$ of $\left(X_{1}, R_{1}\right)$ such that $\left\langle k^{-}, b\right\rangle \notin R^{*}$. As before with $a, b \neq 0$ and, since $\left\{\bar{k}^{-}\right\}^{*}=\left\{\bar{k}^{-}\right\} \cup b, b$ cannot be a limit ordinal. Therefore, $b$ has a successor, $b^{+}$in $R$, and $\left\langle k^{-}, b^{+}\right\rangle \in R^{*}$. Also, $\langle b, k\rangle \in R^{*}$, because, otherwise, by the induction assumption, $k^{-} R^{*} k R^{*} b \Rightarrow\left\langle k^{-}, b\right\rangle \in R^{*}$, which is impossible. Furthermore, by the induction assumption, $k$ is related to $b^{+}$.

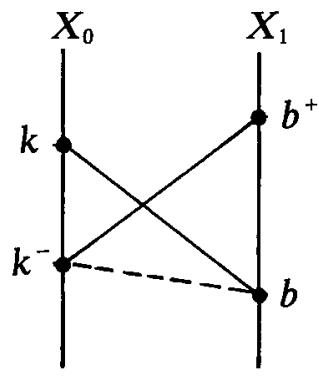

If $\left\langle k, b^{+}\right\rangle \in R^{*}$ and $b$ is unrelated to $k^{-}$, then $k$ covers both $b$ and $k^{-}$ and has no predecessor. If $\left\langle b^{+}, k\right\rangle \in R^{*}$ and $b$ is unrelated to $k^{-}$, then $b^{+}$has no predecessor. Therefore, $b$ is related to $k^{-}$and thus $\left\langle b, k^{-}\right\rangle \in$ $R^{*}$. Then, since $b R^{*} k^{-} R^{*} b^{+}, k^{-}$is related to every element of $X_{1}$. Therefore, by induction, $n$ is related to every element of $X_{1}$ for all $n \leqq p$. 
In particular, $n$ is related to $x$ for all $n \leqq p$ and $x \leqq a$. Therefore, by the same argument as in (a), there is a $t \in X_{1}$ such that $\langle n, t\rangle \in R^{*}$ for all $n \in X_{0}$, and $X_{0} \cup\{\bar{t}\}=\{\bar{t}\}^{*} \in \mathscr{C}^{*} \subseteq \mathscr{C}$, which is impossible.

Thus, there is no $T\left(\delta^{\prime}\right)$-topology $\mathscr{T}^{*}$ on $X$ such that $\mathscr{T} \not \mathscr{T}^{*}$, and $\mathscr{T}$ is a minimal $T\left(\delta^{\prime}\right)$-topology.

\section{REFERENCES}

1. Paul Alexandroff, Sur les espaces discrets, C. R. Acad. Sci. Paris, 200 (1935), 1649-1651.

2. C. E. Aull and W. J. Thron, Separation axioms between $T_{0}$ and $T_{1}$, Indag. Math., 24 (1962), 26-37.

3. Garrett Birkhoff, Lattice Theory, Amer. Math. Soc. Colloquium Publications 25, 3rd. ed., Providence, 1967.

4. K. Császár, Abgeschwächte Trennungsaxiome, General Topology and its Relations to Modern Analysis and Algebra II, Proc. 2nd Prague Topological Symp., 1966, 103-104.

5. A. S. Davis, Indexed systems of neighborhoods for general topological spaces, Amer. Math. Monthly, 68 (1961), 886-893.

6. Jean-Louis Destouches, Les espaces à caractère fini, C. R. Acad. Sci. Paris, 204 (1937), 219-222.

7. Maurice Fréchet, Espaces Abstraits, Paris, 1928.

8. Otto Fröhlich, Das Halbordnungssystem der topologischen Räume auf einer Menge, Math. Ann., 156 (1964), 79-95.

9. Roland E. Larson, Minimal $T_{0}$-spaces and minimal $T_{D}$-spaces, Pacific J. Math., 31 (1969), 451-458.

10. Norman Levine, Strongly connected sets in topology, Amer. Math. Monthly, 72 (1965), 1098-1101.

11. B. A. Linfield, Espace discret paramétrique et non paramétrique, (thesis presented to the University of Strasbourg, July 30, 1925).

12. Teng-Sun Liu, A note on maximal $T_{1}$-topologies, Portugal. Math., 18 (1959), 235-236.

13. Francois Lorrain, Notes on topological spaces with minimum neighborhoods, Amer. Math. Monthly, 76 (1969), 616-627.

14. Oystein Ore, Some studies on closure relations, Duke Math. J., 10 (1943), 761-785.

15. Ki-Hyun Pahk, Note on the characterizations of minimal $T_{0}$ and $T_{\mathrm{D}}$-spaces, Kyungpook Math. J., 8 (1968), 5-10.

16. A. K. Steiner, The lattice of topologies: structure and complementation, Trans. Amer. Math. Soc., 122 (1966), 379-398.

17. J. Pelham Thomas, Maximal connected topologies, J. Austral. Math. Soc., 8 (1968), 700-705.

18. W. J. Thron, Topological Structures, New York, 1966.

19. W. J. Thron and Susan J. Zimmerman, A characterization of order topologies by means of minimal $T_{0}$-topologies, Proc. Amer. Math. Soc., 27 (1971), 161-167.

20. A. W. Tucker, Cell spaces, Ann. of Math., 37 (1936), 92-100.

21. J. W. T. Youngs, $A$ note on separation axioms and their application in the theory of a locally connected topological space, Bull. Amer. Math. Soc., 49 (1943), 383-385.

22. Susan Jane Zimmerman, Order-induced topological properties, (thesis submitted to the University of Colorado, 1970).

Received January 7, 1976. 


\section{PACIFIC JOURNAL OF MATHEMATICS}

\section{EDITORS}

RICHARD ARENS (Managing Editor)

University of California

Los Angeles, CA 90024

R. A. BeAumont

University of Washington

Seattle, WA 98105

C. C. MOORE

University of California

Berkeley, CA 94720
J. DUGUNDJI

Department of Mathematics

University of Southern California

Los Angeles, CA 90007

R. FINN AND J. MILGRAM

Stanford University

Stanford, CA 94305

\section{ASSOCIATE EDITORS}
E. F. BECKENBACH
B. H. NEUMANN
F. WOLF
K. YoshidA

\section{SUPPORTING INSTITUTIONS}

UNIVERSITY OF BRITISH COLUMBIA

UNIVERSITY OF SOUTHERN CALIFORNIA

CALIFORNIA INSTITUTE OF TECHNOLOGY

STANFORD UNIVERSITY

UNIVERSITY OF CALIFORNIA

UNIVERSITY OF HAWAII

MONTANA STATE UNIVERSITY

UNIVERSITY OF TOKYO

UNIVERSITY OF NEVADA

UNIVERSITY OF UTAH

NEW MEXICO STATE UNIVERSITY

OREGON STATE UNIVERSITY

UNIVERSITY OF OREGON

OSAKA UNIVERSITY

WASHINGTON STATE UNIVERSITY

UNIVERSITY OF WASHINGTON

AMERICAN MATHEMATICAL SOCIETY

The Supporting Institutions listed above contribute to the cost of publication of this Journal, but they are not owners or publishers and have no responsibility for its contents or policies.

Mathematical papers intended for publication in the Pacific Journal of Mathematics should be in typed form or offset-reproduced (not dittoed), double spaced with large margins. Underline Greek letters in red, German in green, and script in blue. The first paragraph or two must be capable of being used separately as a synopsis of the entire paper. Items of the bibliography should not be cited there unless absolutely necessary, in which case they must be identified by author and Journal, rather than by item number. Manuscripts, in duplicate, may be sent to any one of the four editors. Please classify according to the scheme of Math. Reviews, Index to Vol. 39. All other communications should be addressed to the managing editor, or Elaine Barth, University of California, Los Angeles, California, 90024.

100 reprints are provided free for each article, only if page charges have been substantially paid. Additional copies may be obtained at cost in multiples of 50 .

The Pacific Journal of Mathematics is issued monthly as of January 1966. Regular subscription rate: $\$ 72.00$ a year (6 Vols., 12 issues). Special rate: $\$ 36.00$ a year to individual members of supporting institutions.

Subscriptions, orders for numbers issued in the last three calendar years, and changes of address should be sent to Pacific Journal of Mathematics, 103 Highland Boulevard, Berkeley, California, 94708.

PUBLISHED BY PACIFIC JOURNAL OF MATHEMATICS, A NON-PROFIT CORPORATION

Printed at Jerusalem Academic Press, POB 2390, Jerusalem, Israel. 


\section{Pacific Journal of Mathematics}

\section{Vol. 75, No. $2 \quad$ October, 1978}

Susan Jane Zimmerman Andima and W. J. Thron, Order-induced

topological properties ................................... 297

Gregory Wade Bell, Cohomology of degree 1 and 2 of the Suzuki groups . . 319

Richard Body and Roy Rene Douglas, Rational homotopy and unique

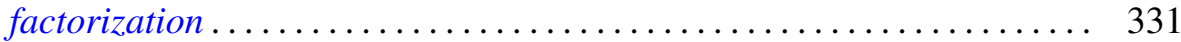

Frank Lewis Capobianco, Fixed sets of involutions ................. 339

L. Carlitz, Some theorems on generalized Dedekind-Rademacher sums .... 347

Mary Rodriguez Embry and Alan Leslie Lambert, The structure of a special class of weighted translation semigroups .....................

Steve Ferry, Strongly regular mappings with compact ANR fibers are

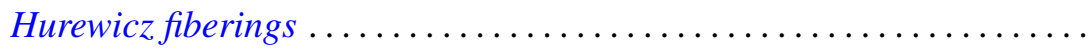

Ivan Filippenko and Marvin David Marcus, On the unitary invariance of the

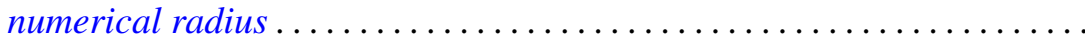

$\mathrm{H}$. Groemer, On the extension of additive functionals on classes of convex

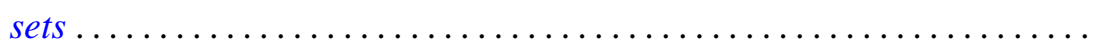

Rita Hall, On the cohomology of Kuga's fiber variety ............... 411

H. B. Hamilton, Congruences on $\mathrm{N}$-semigroups ................. 423

Manfred Herrmann and Rolf Schmidt, Regular sequences and lifting

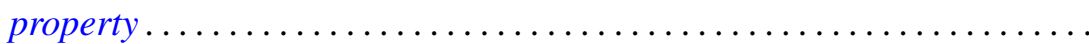

James Edgar Keesling, Decompositions of the Stone-Čech compactification which are shape equivalences .....................

Michael Jay Klass and Lawrence Edward Myers, On stopping rules and the expected supremum of $S_{n} / T_{n}$

Ronald Charles Linton, $\lambda$-large subgroups of $C_{\lambda}$-groups

William Owen Murray, IV and L. Bruce Treybig, Triangulations with the free cell property ............................

Louis Jackson Ratliff, Jr., Polynomial rings and $H_{i}$-local rings ...

Michael Rich, On alternate rings and their attached Jordan rings....

Gary Sampson and H. Tuy, Fourier transforms and their Lipschitz

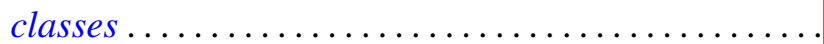

Helga Schirmer, Effluent and noneffluent fixed points on dendrites ...

Daniel Byron Shapiro, Intersections of the space of skew-symmetric maps

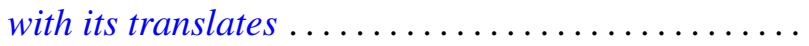

Edwin Spanier, Tautness for Alexander-Spanier cohomology ...

Alan Stein and Ivan Ernest Stux, A mean value theorem for binary digits ...

Franklin D. Tall, Normal subspaces of the density topology . .

William Yslas Vélez, Prime ideal decomposition in $F\left(\mu^{1 / p}\right) \ldots$ 